\title{
A!
}

This is an electronic reprint of the original article.

This reprint may differ from the original in pagination and typographic detail.

Khaymovich, I. M.; Maisi, V. F.; Pekola, J. P.; Mel'nikov, A.S.

\section{Charge-vortex interplay in a superconducting Coulomb-blockaded island}

Published in:

Physical Review B

DOI:

10.1103/PhysRevB.92.020501

Published: 06/07/2015

Document Version

Publisher's PDF, also known as Version of record

Please cite the original version:

Khaymovich, I. M., Maisi, V. F., Pekola, J. P., \& Mel'nikov, A. S. (2015). Charge-vortex interplay in a superconducting Coulomb-blockaded island. Physical Review B, 92(2), 1-5. [020501].

https://doi.org/10.1103/PhysRevB.92.020501

This material is protected by copyright and other intellectual property rights, and duplication or sale of all or part of any of the repository collections is not permitted, except that material may be duplicated by you for your research use or educational purposes in electronic or print form. You must obtain permission for any other use. Electronic or print copies may not be offered, whether for sale or otherwise to anyone who is not an authorised user. 


\title{
Charge-vortex interplay in a superconducting Coulomb-blockaded island
}

\author{
I. M. Khaymovich, ${ }^{1,2,{ }^{*}}$ V. F. Maisi, ${ }^{1,3,4}$ J. P. Pekola, ${ }^{1}$ and A. S. Mel'nikov ${ }^{2,5}$ \\ ${ }^{1}$ Low Temperature Laboratory, Department of Applied Physics, Aalto University, P.O. Box 13500, FI-00076 Aalto, Finland \\ ${ }^{2}$ Institute for Physics of Microstructures, Russian Academy of Sciences, 603950 Nizhni Novgorod, GSP-105, Russia \\ ${ }^{3}$ Solid State Physics Laboratory, ETH Zürich, CH-8093 Zürich, Switzerland \\ ${ }^{4}$ Centre for Metrology and Accreditation (MIKES), P.O. Box 9, FI-02151 Espoo, Finland \\ ${ }^{5}$ Lobachevsky State University of Nizhni Novgorod, 23 Prospekt Gagarina, 603950 Nizhni Novgorod, Russia \\ (Received 2 February 2015; revised manuscript received 27 March 2015; published 6 July 2015)
}

\begin{abstract}
We show that charge transfer through a small superconducting (S) island of a single-electron transistor is strongly affected by vorticity. This interplay of charge and rotational degrees of freedom in a mesoscopic superconductor occurs through the effect of vorticity on the quantum mechanical spectrum of electron-hole excitations. The subgap quasiparticle levels in vortices can host an extra electron, thus suppressing the so-called parity effect in the $\mathrm{S}$ island. We propose to measure the collective dynamics of vorticity and electric charge via the charge pumping effect caused by alternating vortex entry and exit controlled by a periodic magnetic field.
\end{abstract}

DOI: 10.1103/PhysRevB.92.020501

There are two fundamental quantization phenomena which are manifested in different aspects of the physics of superconducting (S) metals: (i) quantization of the charge of superconducting carriers or Cooper pairs in units of double electron charge $2 e$ and (ii) quantization of the trapped magnetic flux in units of the flux quantum $\Phi_{0}=h c / 2 e$, which originates from vorticity quantization [1]. The duality of these quantization effects reveals itself in Coulomb and Weber (or vortex) blockade phenomena, as has been discussed recently in Ref. [2]. In addition to this remarkable duality, it would be certainly exciting to observe the charge-vortex interplay which could cause magnetoelectric phenomena inherent, e.g., to a superconducting state containing topological defects with quantized vorticity, namely, Abrikosov vortices. Unfortunately, for the bulk samples it is extremely difficult to observe these effects experimentally. The reason is that for typical metals the large value of the Fermi energy $E_{F}$ almost completely suppresses all the electrostatic charge phenomena caused by vortices: The vortex core charge is small due to the very small ratio $\Delta / E_{F} \sim 10^{-5}-10^{-2}[3,4]$. Here, $\Delta$ is the superconducting order parameter.

In the present Rapid Communication we make a suggestion on how to overcome the above difficulties based on the powerful methods provided by superconducting single-electron devices (see Refs. [5,6] and references therein). The key point of this idea is that by creating or removing a single vortex in a small $\mathrm{S}$ island, either the odd or even electron number will be favored. Thus the vorticity and the charge of the island will be coupled. To address electrons one by one, we propose to use a single-electron transistor (SET), i.e., a small, metallic Coulomb-blockaded island with a total electric capacitance $C=C_{L}+C_{R}+C_{g}$ (see Fig. 1) coupled to the normal (N) leads by tunnel contacts with capacitances $C_{L}$ and $C_{R}$. A large Coulomb energy $E_{C}=e^{2} /(2 C)$ compared to the temperature $T$ prevents an extra electron from tunneling in and allows one to manipulate the charge state $n$ of the island in a controllable way by varying the gate voltage $V_{g}=e n_{g} / C_{g}$ across the capacitor $C_{g}$. For an $\mathrm{S}$ island this physical picture becomes more complicated due to the electron number parity effect [7-10].

*Corresponding author: ivan.khaymovich@aalto.fi
PACS number(s): 73.23.Hk, 74.25.Ha, 85.35.Gv

This effect consists in the $2 e$-periodic dependence of the observables on $V_{g}$ and thus provides a direct confirmation of the Cooper pair charge quantization. At finite temperatures the parity phenomena are controlled by the free energy difference $\delta F=\Delta-T \ln N_{\text {eff }}$ between the states with an odd and even number of electrons in the island. Here, $N_{\text {eff }}$ is the effective number of available states for an extra particle. It is clear that the parity effect is observable only for a positive value of this free energy barrier, i.e., at low enough temperatures, $T<$ $T^{*}=\Delta / \ln N_{\text {eff. }}$. By applying an external magnetic field $H$ one can partially suppress the $S$ gap and thus affect the parity phenomenon. Appropriate changes of periodicity of the SET characteristics versus the gate voltage in the $H$ field have been observed, e.g., in Ref. [11]. It is important to note, however, that a partial decrease of the gap preceding the vortex entry might not be enough to suppress the parity effect at rather low temperatures (see the gap estimates below). The only guaranteed way to cause a change in the charge state of the $\mathrm{S}$ island is to introduce a vortex, therefore providing a natural trap for an extra electron.

Applying an oscillating magnetic field, i.e., changing periodically the island vorticity, we can induce the even-odd transitions in the number $n$ of electrons in the island. Choosing the gate voltage, as shown in Fig. 2 by a black dot, one can switch between the states $n=0$ and $n=1$. Without the vortex, the state with an even $n=0$, shown by the large white diamond, is favored. With the vortex, the odd electron number becomes preferable, as shown by the dashed red diamonds. Applying a constant bias voltage $V$ to the SET, one can convert this modulation of the charge state into unidirectional charge pumping. Note that another version of magnetic field induced pumping can be realized in the Cooper pair sluices suggested for Josephson structures with S electrodes [12]. The above picture of the vortex controlled parity effect can change at temperatures less than the minigap $\omega_{0} \ll \Delta$ in the spectrum of quasiparticles trapped in the vortex core [13]. The free energy barrier for even-odd transitions in the vortex state can be estimated as $\delta F_{v}=\omega_{0}-T \ln N_{v}$, where $N_{v} \sim k_{F} L$, $L$ is the length of the vortex line, and $k_{F}$ is the Fermi momentum. The temperature $T_{v}^{*}=\omega_{0} / \ln N_{v}$ at which $\delta F_{v}=0$ separates the regimes with $e$ and $2 e$ charge periods and thus at $T<T_{v}^{*}$ the parity effect can be restored. It is useful to note an analogy with the parity effect in the Josephson 


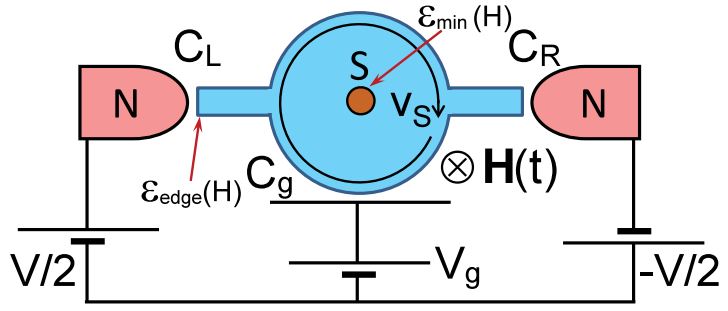

FIG. 1. (Color online) Normal metal-insulator-superconductorinsulator-normal metal (NISIN) SET setup with a bias voltage $\pm V / 2$. The magnetic field is perpendicular to the $\mathrm{S}$ disk plane.

junction [14], where $\omega_{0}$ should be replaced by the minigap depending on the phase difference between the S leads.

We now proceed with the study of an exemplary SET setup (see Fig. 1) which allows us to illustrate the above chargevortex interplay. The size of the $\mathrm{S}$ island is assumed to be of the order of several coherence lengths $\xi$ so that by applying the $H$ field we can introduce at least one vortex in this island. The charge transport through this device can be described by a standard rate equation accounting for parity effects [15]. We restrict ourselves to a two-level approximation assuming $T \ll E_{C}$ and taking the gate voltage interval $0<n_{g}<1$. The equation for the $n=1$ charge state probability $p_{1}$ reads

$$
\frac{d p_{1}}{d t}=\Gamma_{0 \rightarrow 1} p_{0}-\Gamma_{1 \rightarrow 0} p_{1}, \quad p_{0}=1-p_{1},
$$

where $\Gamma_{0 \rightarrow 1}$ and $\Gamma_{1 \rightarrow 0}$ are the rates for the electron tunneling into and out of the island, respectively. $\Gamma_{0 \rightarrow 1}$ and $\Gamma_{1 \rightarrow 0}$ consist of the contributions of the tunneling through the left and right contacts, $\Gamma_{0 \rightarrow 1}=\Gamma_{L}^{e}\left[U_{L}\right]+\Gamma_{R}^{e}\left[U_{R}\right]$ and $\Gamma_{1 \rightarrow 0}=$ $\Gamma_{L}^{o}\left[-U_{L}\right]+\Gamma_{R}^{o}\left[-U_{R}\right]$, where

$$
\Gamma_{j}^{k}[U]=\frac{1}{e^{2} R_{T j}} \int_{-\infty}^{\infty} v_{j}(\varepsilon) f_{N}(\varepsilon-U)\left[1-f_{S}^{k}(\varepsilon)\right] d \varepsilon .
$$

Here, $R_{T j}$ is the resistance of the $j$ th junction, $v_{j}(\varepsilon)$ is the local density of states (LDOS) of the island near the $j$ th junction normalized to its normal state value $v_{N}(0), j=L, R$ stands

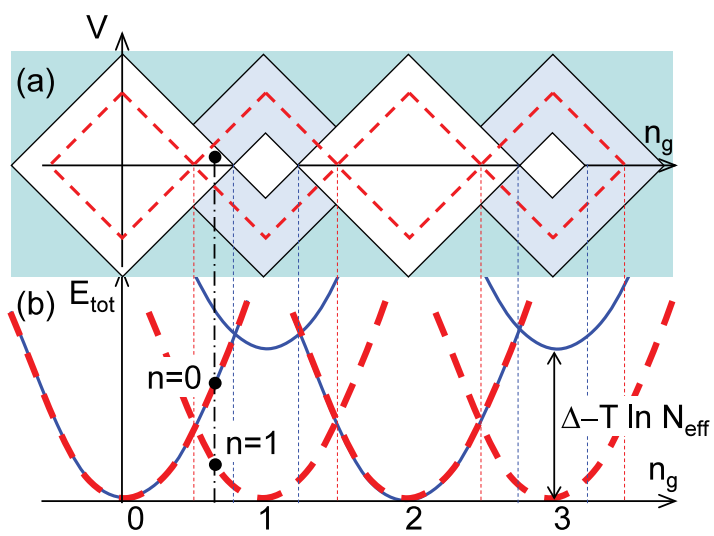

FIG. 2. (Color online) (a) Stability diagram of the S island. Red dashed lines correspond to the normal state stability diagram. The zero current plateaus in the regime of the parity effect are shown by the white diamonds. (b) Ground-state energy of the $\mathrm{S}$ island vs gate voltage with (blue solid lines) and without (red dashed lines) the parity effect. for the left and the right junctions, $f_{N}(\varepsilon)=\left(e^{\varepsilon / T}+1\right)^{-1}$ is the Fermi distribution function in the normal leads, and $f_{S}^{e(o)}(\varepsilon)$ is the distribution function in the $\mathrm{S}$ island describing the states with an even (odd) total number of electrons. The Coulombblockade effect and the bias voltage $V_{L, R}= \pm V / 2$ determine the energy cost $U_{L, R}=E_{C}\left(2 n_{g}-1\right)-e V_{L, R}$ for tunneling.

The increasing magnetic field and vortex entry affect both the LDOS $v_{L, R}(\varepsilon)$ and $f_{S}^{e, o}(\varepsilon)$. To find the distribution function $f_{S}^{e(o)}(\varepsilon)$ we assume that $n=0(n=1)$ corresponds to an even (odd) total number of electrons and use the so-called parity projection technique $[8,16,17]$,

$$
f_{S}^{e(o)}(\varepsilon)=\frac{f_{F}(\varepsilon) \mp \exp \left(-2 N_{\mathrm{qp}}\right) f_{B}(\varepsilon)}{1 \pm \exp \left(-2 N_{\mathrm{qp}}\right)},
$$

where $f_{F(B)}=\left(e^{\varepsilon / T} \pm 1\right)^{-1}$ is the Fermi (Bose) distribution function. The number of quasiparticles is

$$
N_{\mathrm{qp}}=2 v_{N}(0) \int d V \int_{0}^{\infty} v(\varepsilon, \mathbf{r}) f_{F}(\varepsilon) d \varepsilon .
$$

In the limit $f_{F, B} \ll 1$ one can neglect the difference between these distribution functions and reduce (3) to the form [18] $f_{S}^{e, o}(\varepsilon)=A_{e, o} f_{F}(\varepsilon)$ with the factor $A_{e}=A_{o}^{-1}=\tanh \left(N_{\mathrm{qp}}\right)$. In the limit $T \ll \varepsilon_{\min }$, with $\varepsilon_{\min }$ being the minigap in the quasiparticle spectrum of the island, we obtain $N_{\mathrm{qp}} \approx N_{\mathrm{eff}} e^{-\varepsilon_{\min } / T}$, where $N_{\text {eff }}$ is a slow function of temperature $T$ (see the Supplemental Material [19]).

Within the region of the essential parity effect (when $\mid A_{k}$ $1 \mid \sim 1$ ) we can rewrite the tunneling rate as follows:

$$
\begin{aligned}
& \Gamma_{j}^{k}[U>0]=I_{j}(U) / e\left[1+\frac{A_{k}}{e^{U / T}-1}\right], \\
& \Gamma_{j}^{k}[U<0]=\frac{I_{j}(U) / e}{1-e^{-U / T}} A_{k},
\end{aligned}
$$

where the "seed" $I-V$ characteristic of the tunnel junction in the absence of the Coulomb effects is

$$
I_{j}(U)=\int_{-\infty}^{\infty} \frac{v_{j}(\varepsilon)}{e R_{T j}}\left[f_{N}(\varepsilon-U)-f_{N}(\varepsilon)\right] d \varepsilon .
$$

Note that $I_{j}(-U)=-I_{j}(U)$. Further calculations should assume a certain model describing the dependence of the $I-V$ curves $I_{j}(U)$ and the number of quasiparticles $N_{\mathrm{qp}}$ on the applied magnetic field. For the sake of simplicity we consider the system to be symmetric: $v_{L}=v_{R}=v(\varepsilon)$ and $R_{T L}=R_{T R}=R_{T}$. In this case key parameters governing the behavior of the $I-V$ curve, i.e., the minigaps $\varepsilon_{j}$ in the quasiparticle spectrum at the $j$ th junction, are also equal, $\varepsilon_{j}=\varepsilon_{\text {edge }}$. The most important part of $I_{j}(U)$ controlling the charge transfer corresponds to small voltages $\left(U \lesssim \varepsilon_{\text {edge }}\right)$ when the $I-V$ curve reveals the temperature activated behavior,

$$
I_{j}(U) \simeq \frac{T}{e R_{T}} e^{-\left(\epsilon_{\text {edge }}-U\right) / T} \int_{0}^{\infty} v\left(\varepsilon_{\text {edge }}+T x\right) e^{-x} d x .
$$

In the limit $U \gg \varepsilon_{\text {edge }}$ we assume a linear dependence $I_{j}(U)=$ $U / e R_{T}$. Note that here we neglect a low voltage contribution to the current arising from the exponential tail of the residual DOS localized inside the vortex core.

Thus, the basic characteristics of our rate equation are determined by the magnetic field dependence of two energy 
scales: (i) the spectral gap $\varepsilon_{\text {edge }}$ at the junctions and (ii) the minimal gap $\varepsilon_{\min }$ over the island. Considering the setup in Fig. 1, one can see that the energy scale $\varepsilon_{\min }$ is determined by the maximum of the local superfluid velocity $v_{S}$ reached either at the edge of the $S$ disk or in the vortex core. The gap $\varepsilon_{\text {edge }}$ at the junctions is determined by the geometry of the $S$ leads attached to the disk. By adding these $S$ leads one can control the magnetic field effect on the tunneling DOS and parity phenomenon independently and avoid complications caused by the vortex effect on the LDOS at the junctions. Taking, first, the diffusion limit with $\xi \gg \ell$ well exceeding the mean free path $\ell$, we find (see Refs. [20-22])

$$
\varepsilon_{\text {edge }}=\Delta(H)\left(1-\gamma_{H}^{2 / 3}\right)^{3 / 2},
$$

where $\Delta(H)=\Delta(0) e^{-\frac{\pi \gamma_{H}}{4}}, \gamma_{H}=\frac{\hbar\left\langle v_{S}^{2}\right\rangle}{2 D \Delta(H)}, D$ is the diffusion coefficient, $\left\langle v_{S}^{2}\right\rangle=(\pi D H w)^{2} / 3 \Phi_{0}^{2}$, and $w \ll \xi$ is the width of the $\mathrm{S}$ lead. To estimate the energy scale $\varepsilon_{\min }$ we use the same expression (8), substituting $\gamma_{H}=\hbar v_{S}^{2} /[2 D \Delta(H)]$ with the maximum local superfluid velocity $v_{S}$. Assuming the screening effects to be small, i.e., the disk radius $R$ to be smaller than the effective London penetration depth, we get $\max v_{S}=\pi D H R / \Phi_{0}$. In the clean limit $\xi \ll \ell$ we should put $\varepsilon_{\text {min }}=\Delta(H)-\hbar k_{F} v_{S}$, where $\Delta(H) \simeq \Delta(0)\left[1-\alpha H^{2} / H_{c}^{2}\right]$. Here, $H_{c} \sim \Phi_{0} / R \xi$ is the field of complete suppression of the surface barrier for the first vortex entry [23,24] and $\alpha \lesssim 1$ is a numerical factor. As shown in Ref. [25], just before the first vortex entry $\varepsilon_{\min }$ tends to zero in the clean (or moderately disordered $\xi \lesssim \ell$ ) limit and remains finite $\varepsilon_{\min } \sim 0.3 \Delta(0)$ in the dirty limit [24-26]. Thus, in the most common dirty regime, the free energy difference $\delta F$ between the states with an odd and even number of excess electrons in the island remains positive at low temperatures up to the first vortex entry and the parity effect in this case can survive the destructive influence of the magnetic field. This is why vortices are indeed required to arrange the charge transfer governed by the external magnetic field. After the vortex enters, $\varepsilon_{\min }$ equals the minigap $\omega_{0}$ in the clean limit and turns to zero in the dirty regime.

In order to model the vortex induced pumping, we assume the following time dependence of the spectral gaps with the period $\tau=t_{0}+t_{v}$ (see Fig. 3) dictated by the piecewise constant applied magnetic field: $\varepsilon_{\text {edge }}=\varepsilon_{\min }=\Delta(0)$ for $0<$ $t<t_{0}$ and $\varepsilon_{\text {edge }}\left(H_{m}\right)=\Delta_{v}, \varepsilon_{\text {min }}\left(H_{m}\right)=\varepsilon_{0}$ for $t_{0}<t<\tau$. In most experimental situations, the characteristic times of the vortex entry and exit and of quasiparticle relaxation processes are vanishing compared to $t_{v}$ and $t_{0}$ (see the upper bound for the frequency $f=1 / \tau$ below), which means that, for simplicity, we can neglect the details of vortex dynamics and its effect on the electronic distribution function. Changing the value $\varepsilon_{0}$ from 0 to $\omega_{0}$, we have the crossover from the dirty to the clean limit (at $H_{m}>H_{c}$ ). Using the solution of Eq. (1),

$$
p_{1}(t)=p_{1}(0) e^{-\int_{0}^{t} \Gamma_{\Sigma} d t^{\prime}}+\int_{0}^{t} \Gamma_{0 \rightarrow 1} e^{-\int_{t^{\prime}}^{t} \Gamma_{\Sigma} d t^{\prime \prime}} d t^{\prime},
$$

we obtain the current (averaged over the charge states) flowing through the $j$ th junction at time instant $t$,

$$
J_{j}(t)=e\left(\Gamma_{j}^{e}\left[U_{j}(t)\right] p_{0}(t)-\Gamma_{j}^{o}\left[-U_{j}(t)\right] p_{1}(t)\right),
$$

where $\Gamma_{\Sigma}=\Gamma_{0 \rightarrow 1}+\Gamma_{1 \rightarrow 0}$. In the case of the periodic magnetic field protocol $\varepsilon_{\text {edge }}(t+\tau)=\varepsilon_{\text {edge }}(t)$,

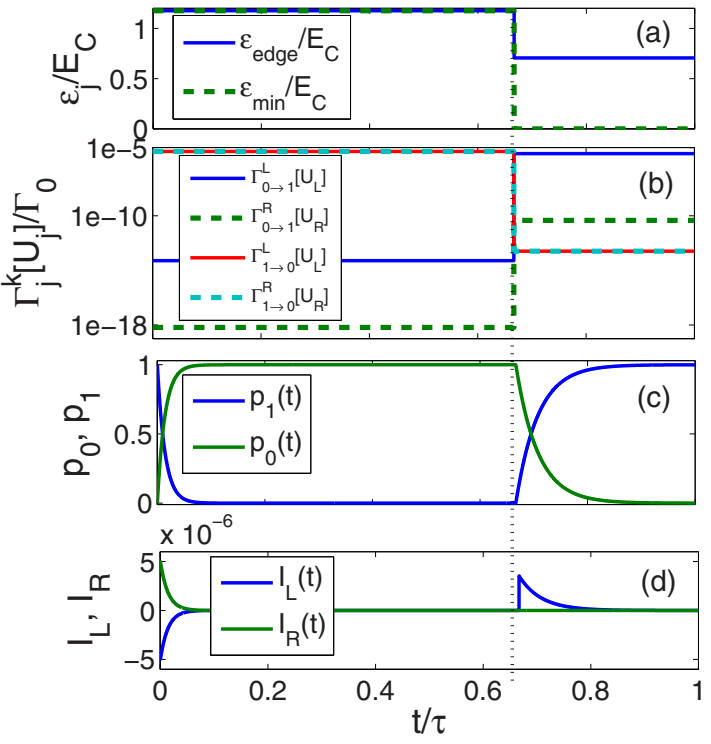

FIG. 3. (Color online) Time dependence of the energy scales $\varepsilon_{\text {edge }}$ and $\varepsilon_{\min }(\mathrm{a})$, the tunneling rates normalized to $\Gamma_{0}(\mathrm{~b})$, the probability distributions (c), and the instantaneous currents through $j$ th junction (d).

$\varepsilon_{\min }(t+\tau)=\varepsilon_{\min }(t)$, the probability distribution arrives at the periodic steady solution after transient processes, when we can impose the condition $p_{1}(0)=p_{1}(\tau)$. Assuming $U_{R}=$ $U_{L}-|e V|>0, U_{L}<\Delta_{v}$, we simplify the expressions for the tunneling rates (5),

$$
\begin{aligned}
& \Gamma_{L, R}^{e} \approx \Gamma_{0} e^{-\left[\varepsilon_{\mathrm{edge}}(t)-U_{L, R}\right] / T}, \\
& \Gamma_{L, R}^{o} \approx \frac{\Gamma_{0} e^{-\varepsilon_{\mathrm{edge}}(t) / T}}{\tanh \left(N_{\mathrm{eff}} e^{-\varepsilon_{\min }(t) / T}\right)},
\end{aligned}
$$

where we omit a slow time dependence of the parameter $\Gamma_{0}=\int_{0}^{\infty} v\left(\varepsilon_{\text {edge }}+\varepsilon\right) e^{-\beta \varepsilon} d \varepsilon / e R_{T}$ [see Eq. (7)]. With these expressions we find the current averaged over the period $\tau$,

$$
\begin{aligned}
\langle J\rangle= & \frac{1}{\tau} \int_{0}^{\tau} J_{j}(t) d t \simeq \frac{e f}{2}\left[1-2 e^{-|e V| / T}+\gamma_{0} t_{0}\right. \\
& \left.+\gamma_{v} t_{v}-e^{-\Gamma_{\Sigma}^{0} t_{0}}-e^{-\Gamma_{\Sigma}^{v} t_{v}}\right],
\end{aligned}
$$

shown in Figs. 4(a) and 4(b) versus bias voltage $V$ and the magnetic field amplitude $H_{m}$. Here, the total tunneling rates $\Gamma_{\Sigma}^{v}$ and $\Gamma_{\Sigma}^{0}$ in the vortex and Meissner states are determined mostly
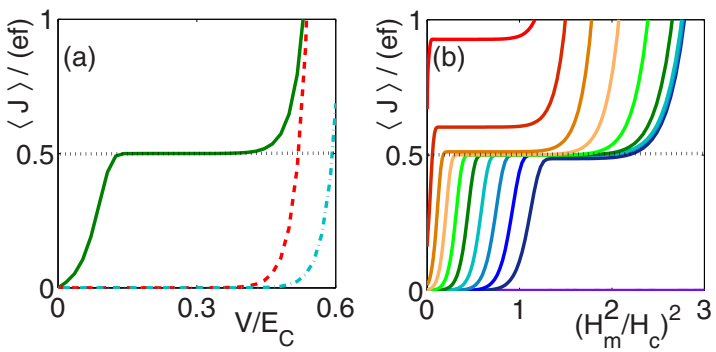

FIG. 4. (Color online) (a) Averaged current $\langle J\rangle$ vs $V$ for $H_{m}=$ $H_{c}$ (green solid line) and $I-V$ curves at $H=0$ (red dashed) and $H=H_{m}$ (blue dashed dotted). (b) The current $\langle J\rangle$ vs the amplitude $H_{m}$ at $V=0 \ldots 0.5 E_{C}$ from left to right. In all plots we put $n_{g}=0.64$. 
by the maximal rates $\Gamma_{\Sigma}^{0} \approx 2 \Gamma_{0} / N_{\mathrm{eff}}, \Gamma_{\Sigma}^{v} \approx \Gamma_{0} e^{-\left(\Delta_{v}-U_{L}\right) / T}$, while $\gamma_{0}=\Gamma_{0} e^{-\left(\Delta-U_{L}\right) / T}, \gamma_{v}=\frac{2 \Gamma_{0} e^{-\Delta_{v} / T}}{\tanh \left(N_{\text {eff }} e^{-\varepsilon_{0} / T}\right)}$ are the small leakage rates. The first two terms in the above expression can be obtained from the following reasoning. When the vortex enters the $\mathrm{S}$ island, the total charge transmitted through both junctions should be equal to the electron charge. Due to the large ratio $\Gamma_{L}^{e} / \Gamma_{R}^{e}=e^{|e V| / T} \gg 1$ of the tunneling rates, most of this charge transfer $e(1-\exp (-|e V| / T))$ occurs through the left junction, while only the exponentially small part of it $\sim e \exp (-|e V| / T)$ is transmitted through the right junction. The vortex exit should be accompanied by the discharge of the island which occurs with equal rates through both junctions (11b). As a result, half of the electron charge exits the island through each junction. Summing up the total charge transmitted through the system per cycle, we find $Q=$ $e\left(1 / 2-e^{-|e V| / T}\right)$. The above symmetry of the discharging processes results in a rather strong shot noise in the system: The fluctuating transmitted charge equals $e / 2$ and the resulting current noise is given by the expression $\sqrt{\left\langle\delta J^{2}\right\rangle}=e f / 2$. The last two terms in (12) appear if the time intervals of two stages $t_{v}$ and $t_{0}$ (with and without a vortex, respectively) become comparable or shorter than the characteristic charging times $1 / \Gamma_{\Sigma}^{v}$ and $1 / \Gamma_{\Sigma}^{0}$. Therefore, the maximum operation frequency $f=1 / \tau$ is limited by a single quasiparticle tunneling rate $\Gamma_{0} / N_{\text {eff }}$. Besides the effect of the frequency, the average current $\langle J\rangle$ also deviates from ef $/ 2$ at small bias voltages and/or small magnetic field amplitudes, due to the dependence of total rates $\Gamma_{\Sigma}^{k}$ on these parameters (see Fig. 4). The terms proportional to $\gamma_{0}$ and $\gamma_{v}$ originate from the leakages and lead to currents exceeding ef $/ 2$, shown at Fig. 4 for larger $V$ and/or $H_{m}$ values. Nevertheless, by satisfying the conditions

$$
\begin{aligned}
N_{\text {eff }} / \Gamma_{0} & \ll t_{0} \ll \gamma_{0}^{-1}, \\
e^{\left(\Delta_{v}-U_{L}\right) / T} / \Gamma_{0} & \ll t_{v} \ll \gamma_{v}^{-1},
\end{aligned}
$$

one can obtain the plateau of the average current at ef/2. These conditions can be met provided we set $e V \gg T$,

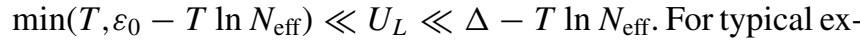
perimental parameters for $\mathrm{Al}$ (see, e.g., Ref. [15]) it gives us a reasonable range of times $0.06 \lesssim t_{0, v} \lesssim 5 \mathrm{~ms}$ in a rather wide range of bias voltages $0.1<|e V| / \Delta<0.6$ to reach a few percent accuracy of the current quantization ef $/ 2$ of a few fA (see the Supplemental Material [19]). In our estimates we used the typical operating temperatures $T \sim 50 \mathrm{mK}$ and sample sizes $R \sim 1 \mu \mathrm{m}$ corresponding to simply accessible field values $H_{m} \sim H_{c} \sim 10 \mathrm{mT}$. The breakdown of the conditions (13) at the lower bound of $U_{L}$ can signal the presence of a minigap in the vortex core in the clean limit $\varepsilon_{0}=\omega_{0}$. Note that for a nonsymmetric setup, the resulting average current will deviate from ef $/ 2$ and the current plateau position should range between 0 and $e f$.

To sum up, we have studied the interplay between vorticity and electric charge which can manifest in conditions of a Coulomb blockade through a vortex induced suppression of the parity effect in mesoscopic samples. Vortex entry and exit from the sample is shown to be accompanied by synchronized entry and exit of a single-electron charge. By applying the bias voltage and oscillating magnetic field one can observe a vortex governed turnstile phenomenon: The switching between the Meissner and vortex states periodically opens the device for single charge transfer. Thus, we have demonstrated that the SET devices provide a unique tool for manipulating the collective dynamics of charge and vorticity. Note that similar devices can be used for detecting heat pulses or photons which suppress the parity effect. The threshold photon frequency in such devices can be tuned by an applied magnetic field.

We are grateful to D. Yu. Vodolazov for useful comments. This work has been supported in part by Academy of Finland (Project No. 284594 and No. 272218), by the European Union Seventh Framework Programme INFERNOS (FP7/2007-2013) under Grant Agreement No. 308850, by the Russian Foundation for Basic Research, and the grant of the Russian Ministry of Science and Education No. 02.B.49.21.0003.
[1] Here we use a standard definition of the vorticity of a certain area as the integral of the gradient of the superconducting phase taken over the contour enclosing this area.

[2] D. Pekker, G. Refael, and P. M. Goldbart, Phys. Rev. Lett. 107, 017002 (2011).

[3] G. Blatter, M. Feigel'man, V. Geshkenbein, A. Larkin, and A. van Otterlo, Phys. Rev. Lett. 77, 566 (1996).

[4] D. I. Khomskii and A. Freimuth, Phys. Rev. Lett. 75, 1384 (1995).

[5] D. V. Averin and K. K. Likharev, J. Low Temp. Phys. 62, 345 (1986); in Mesoscopic Phenomena in Solids, edited by B. L. Altshuler, P. A. Lee, and R. A. Webb, Modern Problems in Condensed Matter Science, Vol. 30 (North-Holland, Amsterdam, 1991), p. 173; in Single Charge Tunneling, edited by H. Grabert and M. H. Devoret, NATO ASI Series Vol. 294 (Plenum, New York, 1992), pp. 217-247.

[6] J. P. Pekola et al., Rev. Mod. Phys. 85, 1421 (2013).
[7] D. V. Averin and Yu. V. Nazarov, Phys. Rev. Lett. 69, 1993 (1992); Physica B 203, 310 (1994).

[8] M. T. Tuominen, J. M. Hergenrother, T. S. Tighe, and M. Tinkham, Phys. Rev. Lett. 69, 1997 (1992).

[9] P. Lafarge, P. Joyez, D. Esteve, C. Urbina, and M. H. Devoret, Phys. Rev. Lett. 70, 994 (1993).

[10] T. M. Eiles, J. M. Martinis, and M. H. Devoret, Phys. Rev. Lett. 70, 1862 (1993).

[11] M. T. Tuominen, J. M. Hergenrother, T. S. Tighe, and M. Tinkham, Phys. Rev. B 47, 11599(R) (1993).

[12] S. Gasparinetti and I. Kamleitner, Phys. Rev. B 86, 224510 (2012).

[13] C. Caroli, P. G. de Gennes, and J. Matricon, Phys. Lett. 9, 307 (1964).

[14] S. V. Sharov and A. D. Zaikin, Phys. Rev. B 71, 014518 (2005).

[15] V. F. Maisi, S. V. Lotkhov, A. Kemppinen, A. Heimes, J. T. Muhonen, and J. P. Pekola, Phys. Rev. Lett. 111, 147001 (2013). 
[16] B. Janko, A. Smith, and V. Ambegaokar, Phys. Rev. B 50, 1152 (1994).

[17] D. S. Golubev and A. D. Zaikin, Phys. Lett. A 195, 380 (1994).

[18] A. Heimes, V. F. Maisi, D. S. Golubev, M. Marthaler, G. Schön, and J. P. Pekola, Phys. Rev. B 89, 014508 (2014).

[19] See Supplemental Material at http://link.aps.org/supplemental/ 10.1103/PhysRevB.92.020501 for details of derivations and for estimates of experimental parameters.

[20] S. Skalski, O. Betbeder-Matibet, and P. R. Weiss, Phys. Rev. 136, A1500 (1964).
[21] K. Maki and P. Fulde, Phys. Rev. 140, A1586 (1965).

[22] A. Anthore, H. Pothier, and D. Esteve, Phys. Rev. Lett. 90, 127001 (2003)

[23] A. I. Buzdin and J. P. Brison, Phys. Lett. A 196, 267 (1994).

[24] P. Fulde, Phys. Rev. 137, A783 (1965).

[25] J. Sanchez-Canizares and F. Sols, J. Low Temp. Phys. 122, 11 (2001).

[26] D. Y. Vodolazov, F. M. Peeters, T. T. Hongisto, and K. Yu. Arutyunov, Europhys. Lett. 75, 315 (2006). 